\title{
las excavadoras de rueda de cangilones en los grandes movimientos de tierra
}

G. VIE, Ingeniero de Minas

sinopsis

El autor hace una breve historia de este tipo de máquinas y habla del considerable progreso que han experimentado en los últimos 30 años, en cuanto a tamaño, peso y eficacla, añadiendo que los más recientes proyectos tienden a colocarlas en situación privilegiada, sin aparente com
cidad de rendimiento y de ataque a formaciones de la máxima dureza. En el artículo se describen también las partes de que se componen estas excavadoras, explicando su funcio
namiento, fabricantes, tipos y características más destacadas.

El problema planteado a la maquinaria de movimientos de tierra parece quedar resuelto con la consecución de un dispositivo que trabaje de forma continua, a gran velocidad, y en el cual sean independientes las funciones de excavación y de transporte.

Ya hacia 1900 se patentó una idea con vistas a la realización de una rueda dotada de cuatro palas-cangilones en forma de álabes alargados y fijos a un eje horizontal.

Estos álabes, inspirados sin duda en la ancestral rueda hidráulica de los faraones, desagregaban los materiales que iban a pasar cerca del eje, donde un dispositivo de transporte auxiliar aseguraba la continuidad.

Posteriormente, estos álabes se prolongaron mediante unas canaletas destinadas a guiar los productos de la excavación; sin embargo, su evacuación no quedaba resuelta.

Las actuales excavadoras de rueda de cangilones no son más que una variación de las antiguas creaciones, donde una cinta transportadora reemplaza a las canaletas de evacuación de los álabes.

Debido al aumento del consumo de lignito del valle del Rin, en Alemania, ocasionado por la guerra de 19141918 , los ingenieros de este país trataron de sustituir las excavadoras de "rosario de cangilones", cuyo rendimiento resultaba insuficiente, por una máquina más potente, que sería la rueda de cangilones.

Con esta idea, la firma Humboldt proporcionó en 1916 a los explotadores de Braunkohle, el primer aparato completo.

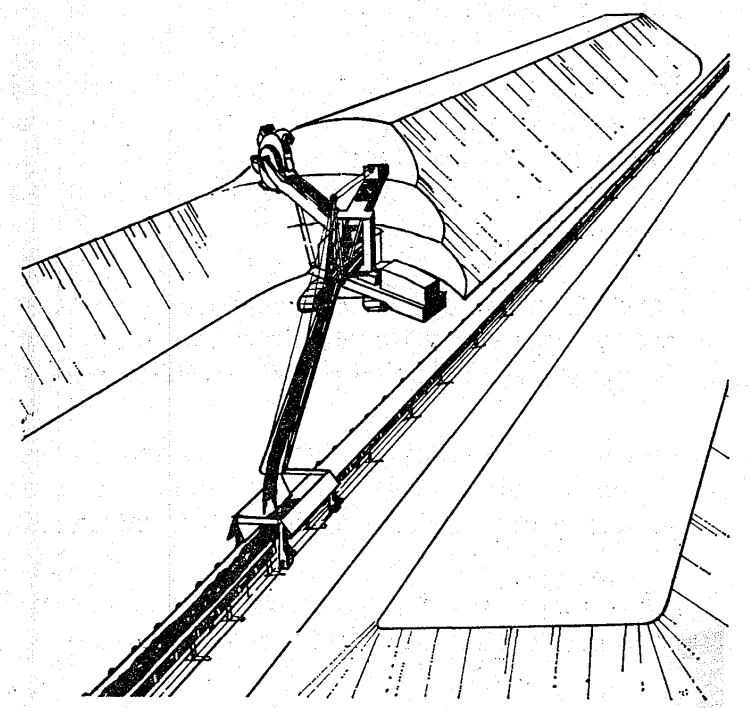

Rueda de cangilones para la retirada de materiales alma cenados. Ejemplo: fosfatos. 


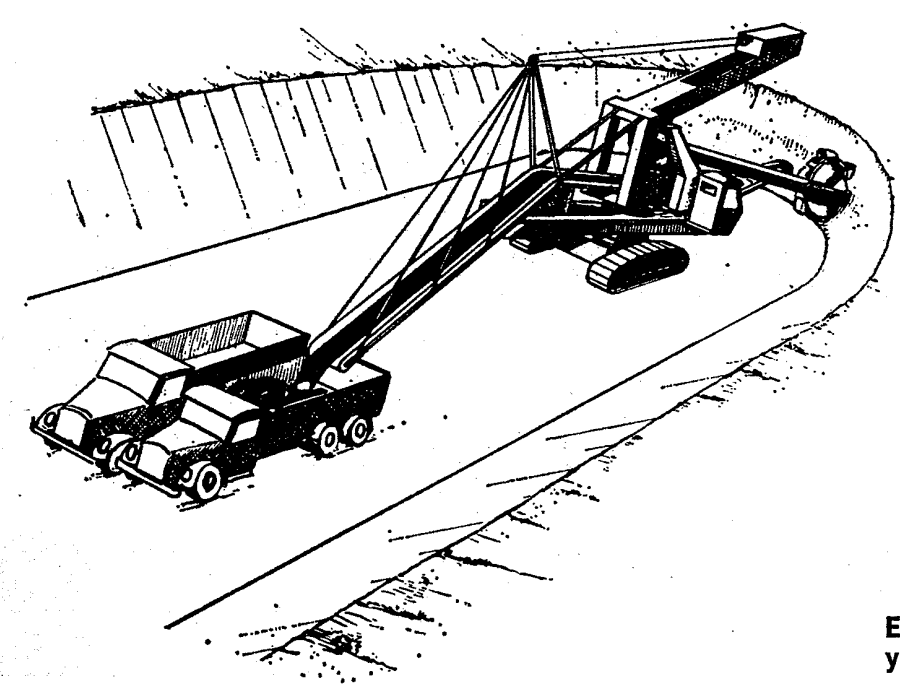

Excavaciones en trinchera de $\mathbf{9 , 5} \mathrm{m}$ de profundidad y de $14 \mathrm{~m}$ de anchura.

En servicio ordinario las averías fueron numerosas, debido a la rotura de piezas, ya que la construcción general de la máquina, con montaje sobre vías, estaba muy débilmente calculada.

En 1923, conseguidos ciertos avances, el prototipo fue enviado a la chatarra. Se fue progresivamente hacia la máquina automotriz, aunque con una robustez todavía insuficiente.

Tras no pocas vicisitudes, el año 1934 se puede considerar como el período de su perfecta puesta a punto, con un desarrollo excepcional, pero con dimensiones menores que las de las grandes máquinas actuales.

En Francia, a partir de 1930, se construyó una rueda de cangilones siguiendo los cálculos del ingeniero Ries, en aquella fecha director de la Societé Minière et Electrique des Landes, y puesta en servicio en Hostens (a $40 \mathrm{~km}$ al sur de Burdeos), para la extracción de tierras de recubrimiento y del lignito necesario para la alimentación de las calderas de la central térmica de Hostens.

La excavadora estaba suspendida de un pórtico y su construcción debía soportar grandes esfuerzos, ya que el lignito era de difícil extracción a causa de los importantes volúmenes de cepas y troncos de árboles exóticos fósiles existentes.

Esta máquina, de funcionamiento satisfactorio, ha estado en servicio alrededor de 30 años. La capacidad inicial se pudo doblar aumentando la potencia del motor, el número y volumen de los cangilones, y la velocidad de las cintas transportadoras.

La rueda inicial tenía un diámetro de $5,50 \mathrm{~m}$ y llevaba seis cangilones de $0,430 \mathrm{~m}^{3}$, girando a 5 r.p.m., arrastrada por un motor de $50 \mathrm{~kW}$.

El rendimiento horario, en lignito, llegó a $100 \mathrm{t}$, ó $390 \mathrm{~m}^{3}$ de tierras de arcilla de recubrimiento. Las transformaciones realizadas llevaron a conseguir el número de nueve cangilones, aunque con una capacidad menor, de $0,200 \mathrm{~m}^{3}$, y a una velocidad de rotación de 7 r.p.m., con un motor de 80-100 kW. 
Ejemplo de explotación en loma con una altura de frente de $9,5 \mathrm{~m}$, y evacuación de los materiales por cinta transportadora.

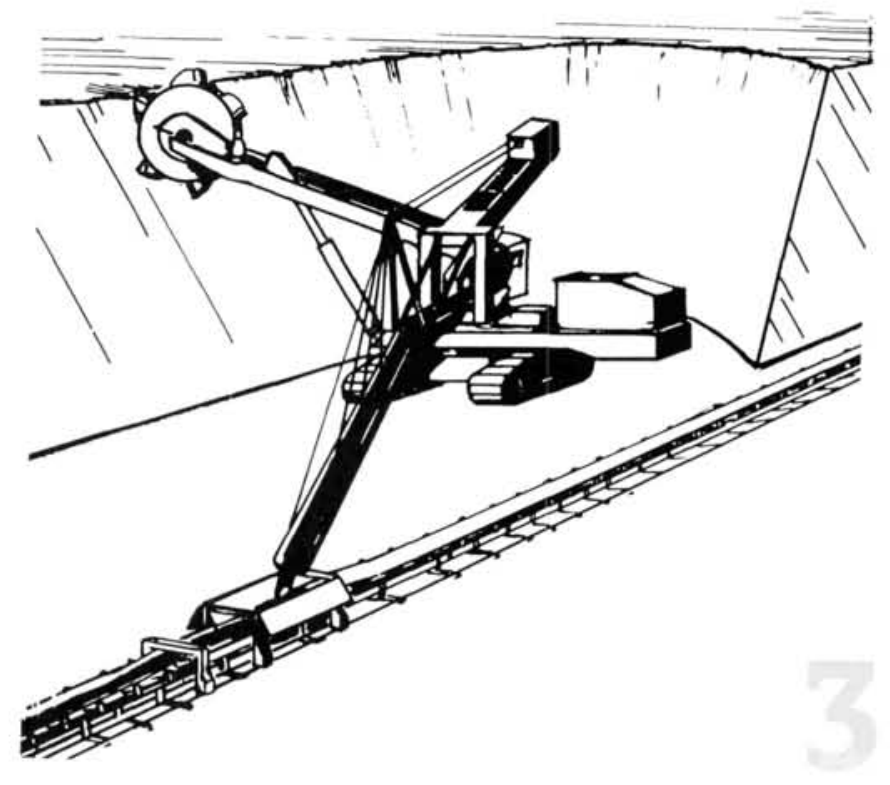

En suma, los rendimientos teóricos pasaron a $250 \mathrm{t} / \mathrm{h}$ para el lignito y 450 a $500 \mathrm{~m}^{3}$ para las tierras estériles superficiales.

Los progresos han sido considerables en el curso de los últimos 30 años, en cuanto al tamaño, peso y producción de la máquina. Los modelos se han complicado para atender a las condiciones particulares de cada caso y de cada yacimiento.

En particular, la generalización de los sistemas hidráulicos ha hecho posible tanto la construcción de máquinas más potentes y capaces, como la extracción de materiales cada vez más duros.

Sin embargo, su campo de acción queda limitado a los materiales relativamente blandos y friables.

A este tipo de maquinaria se le reprocha el inconveniente de la falta de agilidad, pero queda compensada con las ventajas de tipo económico.

Se están estudiando nuevos progresos en la construcción de estas máquinas, que serán sin duda capaces de atacar formaciones más duras, y con rendimientos horarios absolutamente inaccesibles a cualquier otro tipo de maquinaria.

La excavación ejecutada de forma continua proporciona el máximo rendimiento con un esfuerzo mínimo, y el útil de excavación está actuando constantemente; mientras que en una excavación discontinua el útil (cuchara en el caso de pala mecánica y cubeta en caso de dragalina) debe asegurar además, hasta el lugar de evacuación, el transporte de los materiales excavados en el frente de ataque.

De lo expuesto podemos llegar a la conclusión que es necesario disponer de útiles de excavación, de mayores dimensiones, capaces para cargas más voluminosas, con mayor fuerza de arranque y excavación. 


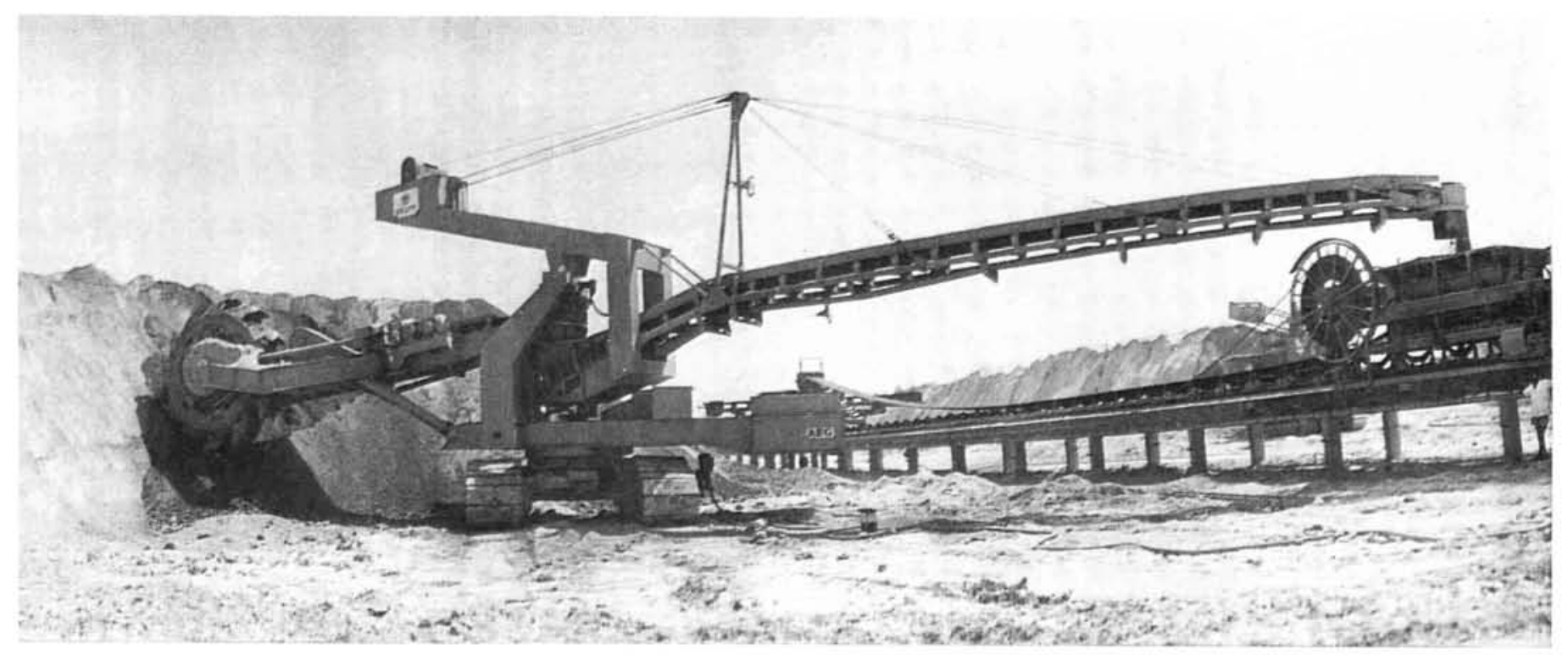

Rueda de cangilones de 390 a $690 \mathrm{~m}^{3} /$ hora en el parque de almacenaje y retirada en los FOSFATOS del TOGO.

Salvando todas las proporciones, y comparativamente a los rendimientos de diferentes máquinas, es evidente la economía de energía a favor de las excavadoras de rueda de cangilones, que podemos cifrar en un $20 \%$.

Una excavadora de rueda de cangilones comprende:

1. Infraestructura sobre orugas.

2. Superestructura orientable con brazo portador de la rueda, regulable en el plano vertical, así como el brazo del contrapeso.

3. Flecha de rechazo con el dispositivo de evacuación de tierras.

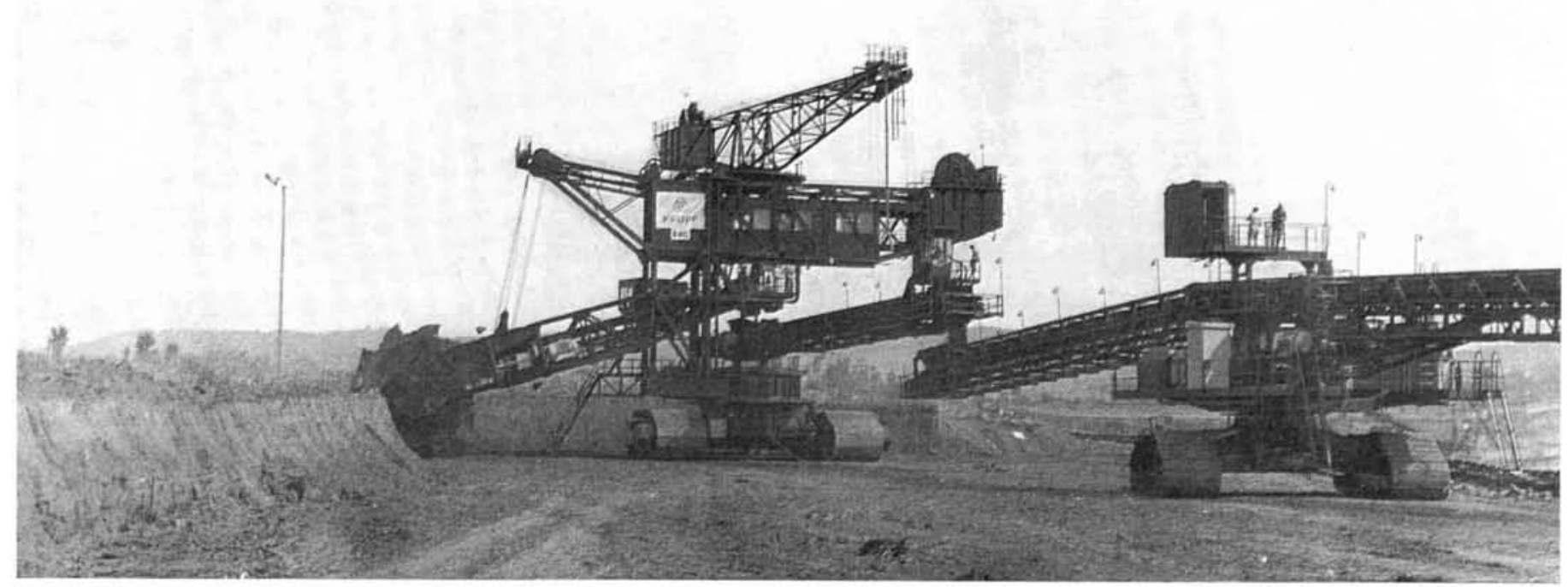

Dos excavadoras semejantes en TRASIMENO y SANTA

BARBARA (lignitos en Italia). 


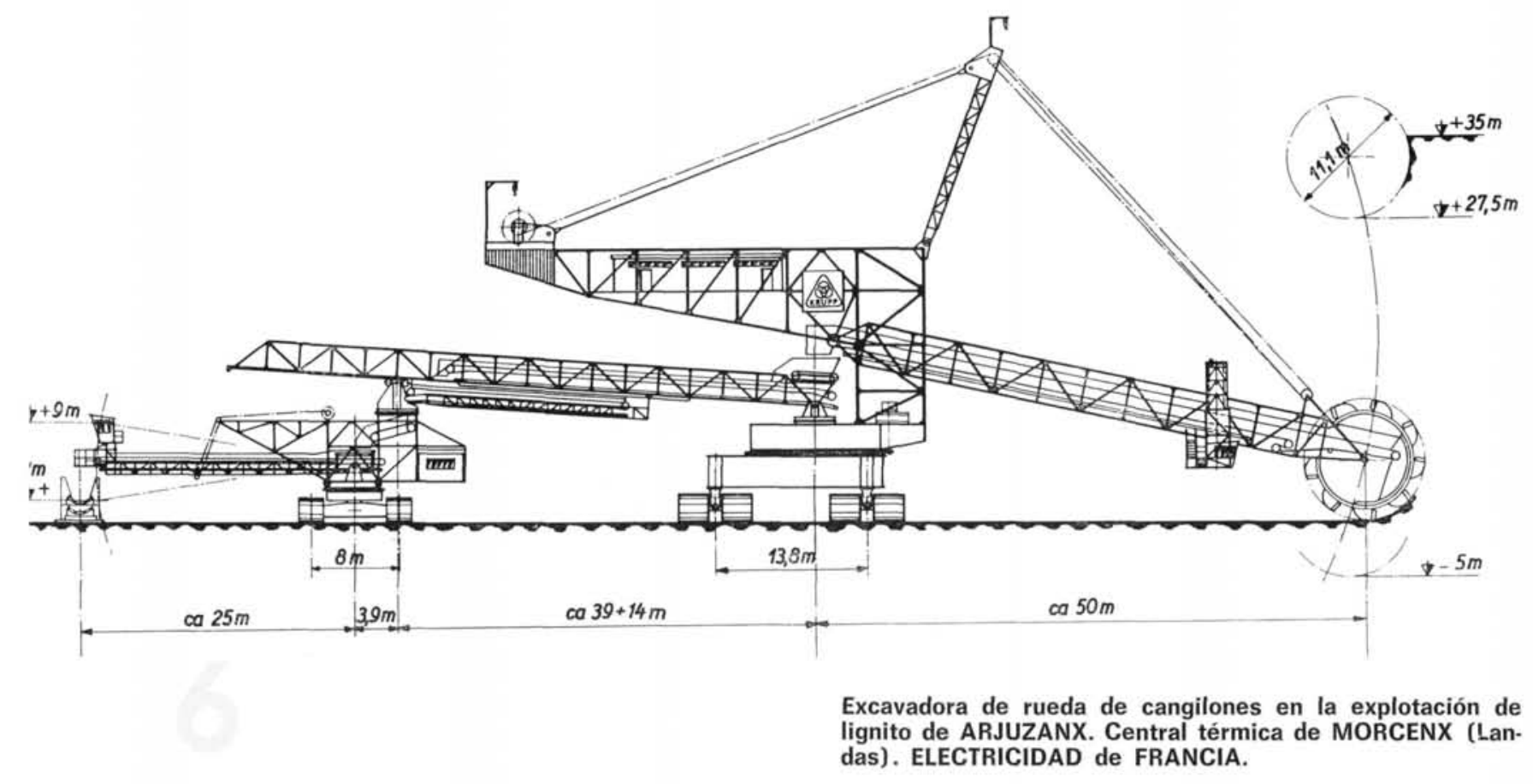

El brazo porta-rueda es orientable y puede ser elevado o abatido, lo que determina la altura del corte del terreno a excavar.

El mecanismo de traslación sobre orugas está unido por tres puntos a la infraestructura.

Si las condiciones del terreno son desfavorables, la suspensión de este tipo se opone a la trans. misión de esfuerzos anormales sobre una de las orugas o sobre la infraestructura.

Por esta razón los rodillos de arrastre de los mecanismos de traslación $y$, en el caso de que éstos sean de orugas múltiples, las orugas están equilibradas conectadas una con otra.

Las máquinas más importantes admiten de 6 a 12 cadenas.

Los eslabones o "tejas" de las orugas están adaptados a las condiciones del suelo, con presión específica media ampliamente calculada $\left(1,2\right.$ a $\left.1,3 \mathrm{kp} / \mathrm{cm}^{2}\right)$ para la traslación.

Esta carga, relativamente débil para el suelo, justifica las importantes dimensiones de los mecanismos de traslación, que pueden alcanzar hasta el $30 \%$ del peso total de la máquina.

Se tendrá, por ejemplo, una superficie portante de $370 \mathrm{~m}^{2}$, repartida en 12 cadenas.

Cuando el brazo porta-rueda deba tener un avance longitudinal, se construye de tipo telescópico.

El brazo de descarga es igualmente orientable y regulable en altura, para poder asegurar, en cualquier posición de la máquina, el vertido de tierras o materiales sobre las cintas transportadoras, vagones y otros vehículos.

Los movimientos de orientación del brazo portador de la rueda y del brazo de descarga son independientes. 


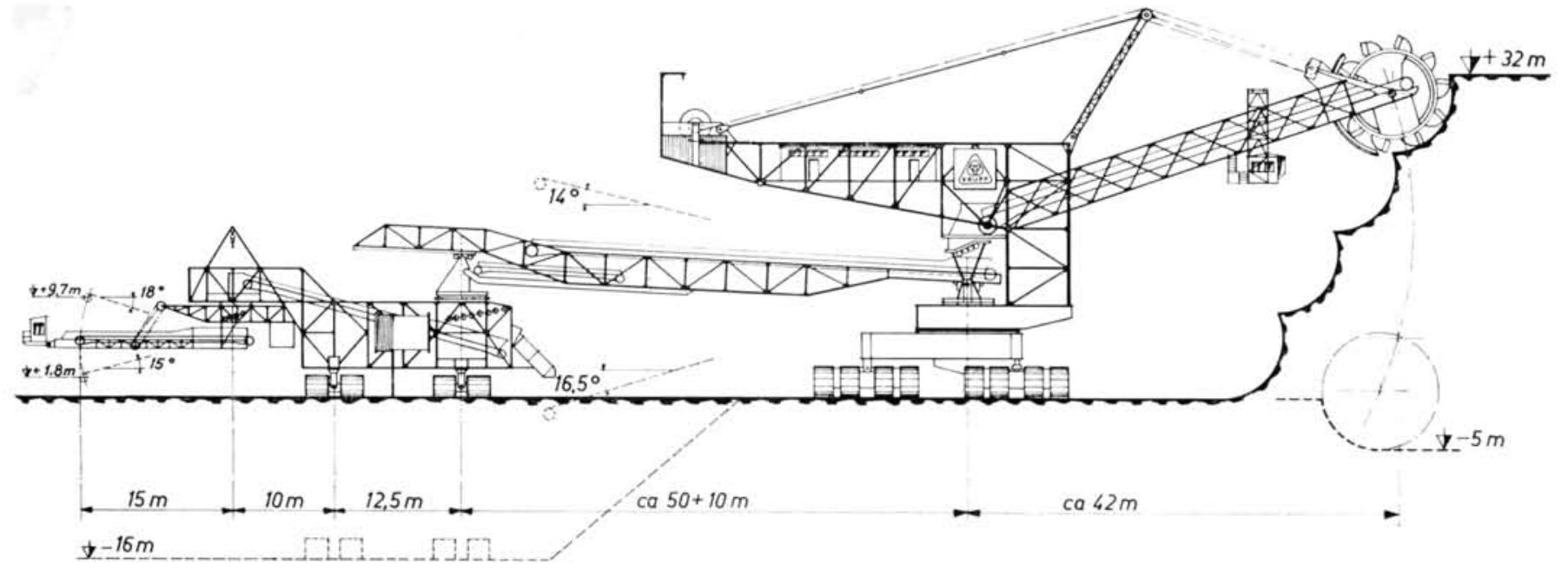

Rheinische Braunkohle A. G., Colonia, sobre el Rin.

Rendimiento: $5.270 \mathrm{~m}^{3} /$ hora.

Minas de FRIMMERSDORF y ZUKUNFT.

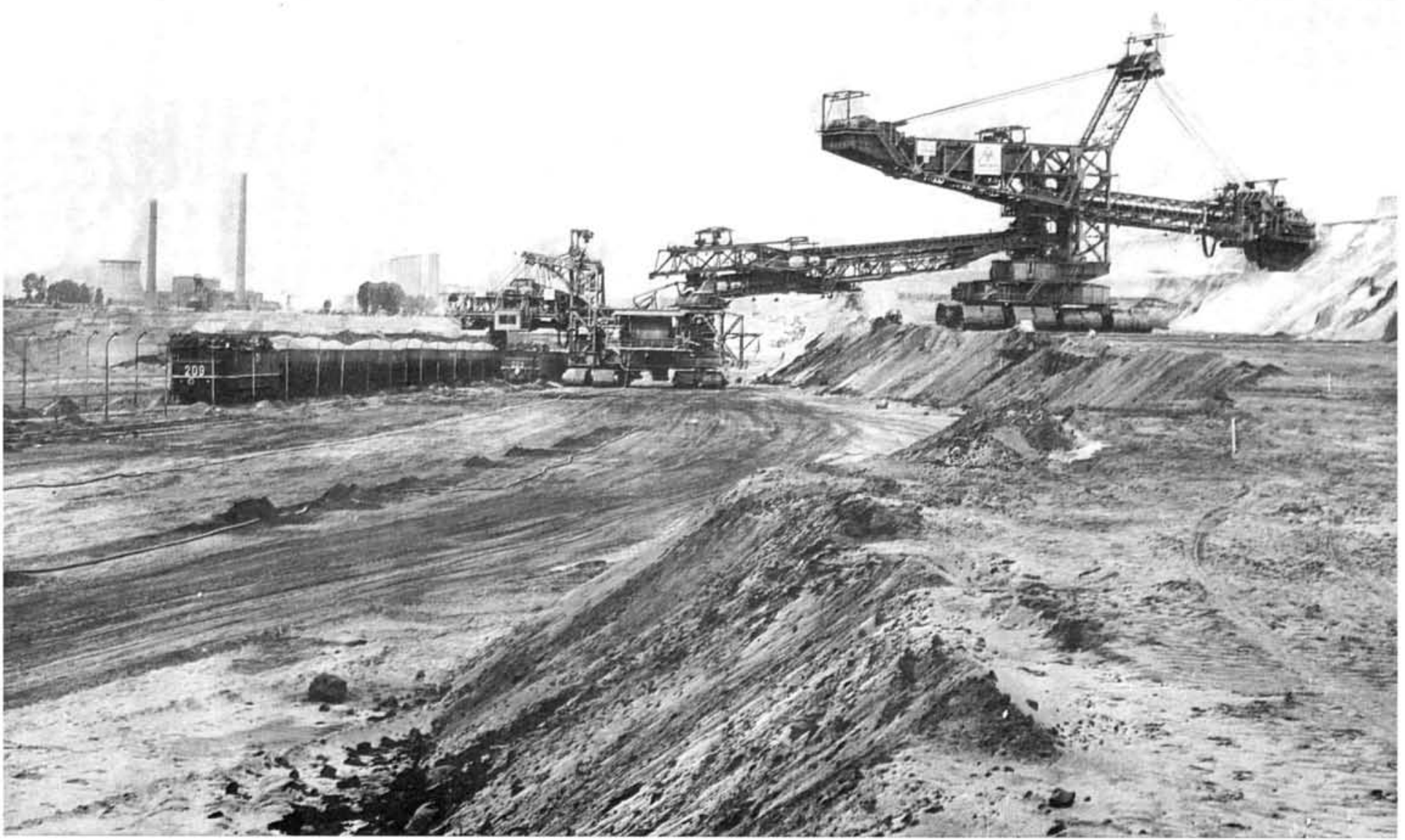

Rheinische Braunkohle A. G., Colonia, sobre el Rin

Excavadora en las minas de FRIMMERSDORF y ZUKUNFT. 


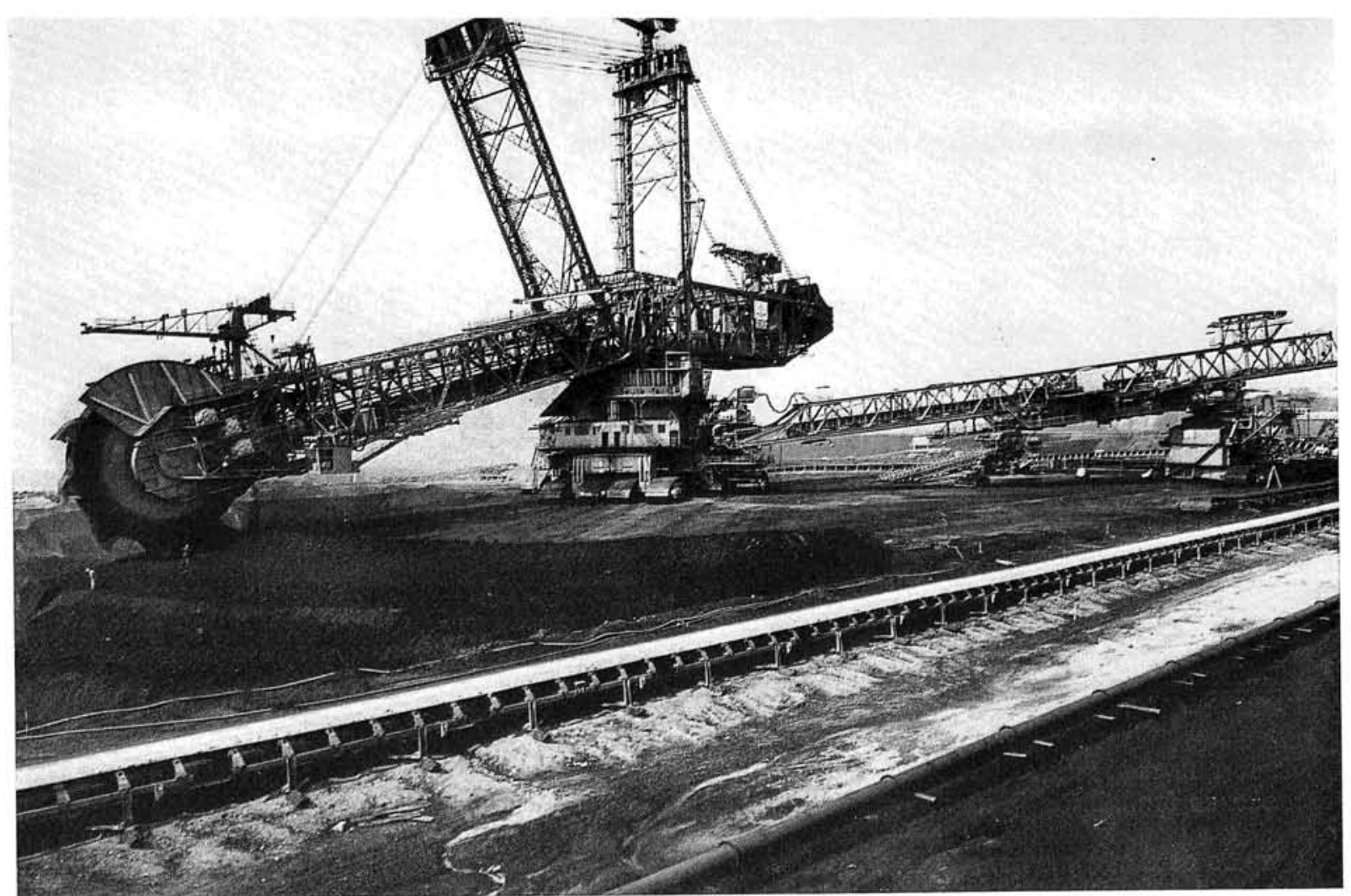

Excavadora de rueda de cangilones $\left(112.000 \mathrm{~m}^{3} / \mathrm{dia}\right.$ de terreno blando). GASDORF (R.F.A.).

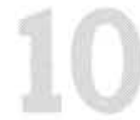

Flecha porta-rueda con su rueda de cangilones. Mina de GASDORF (cuenca de lignitos de Colonia). El conjunto se puede apreciar en la figura anterior.

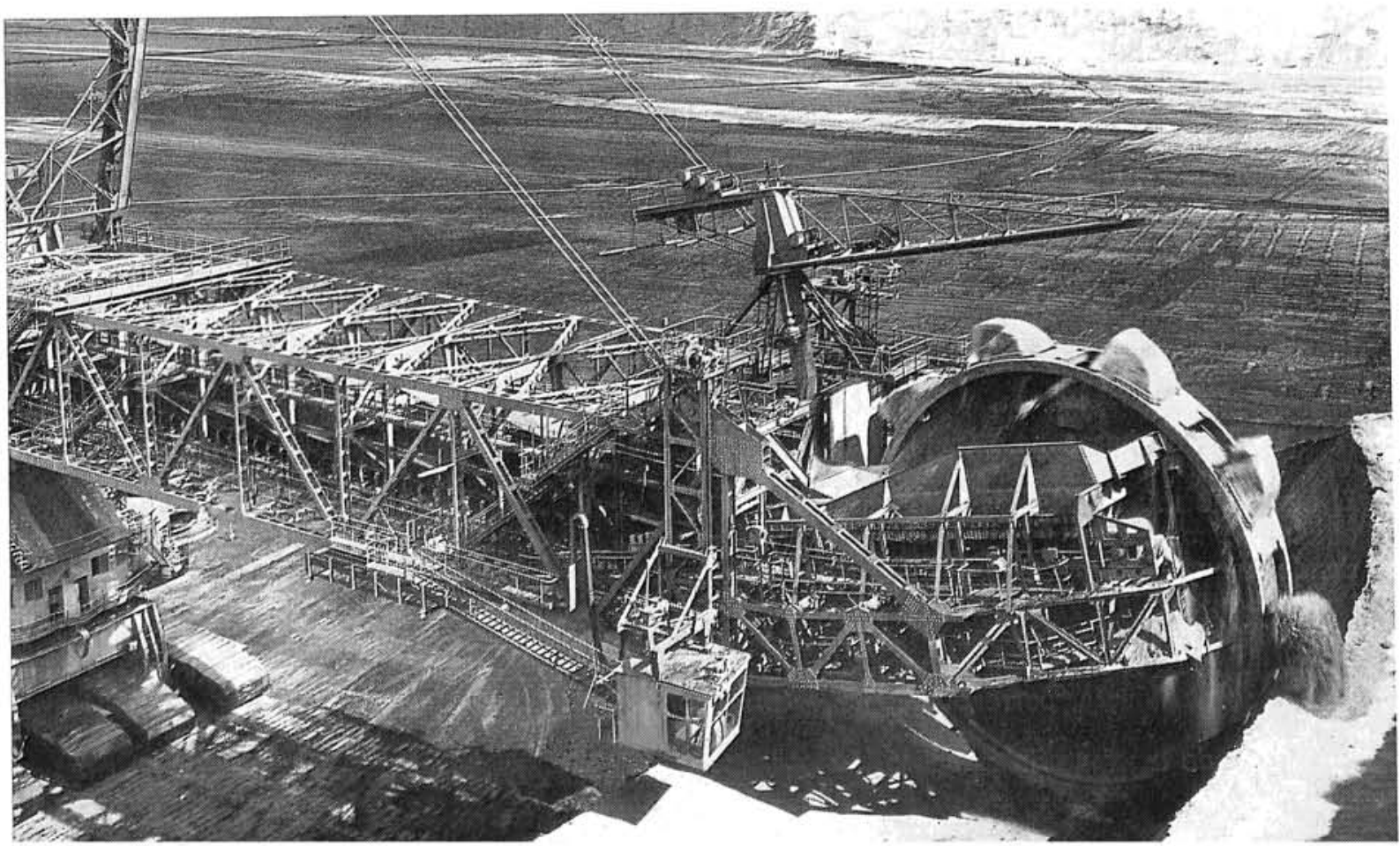


Si la producción horaria alcanza un valor elevado, el dispositivo de descarga se instala sobre un carro autónomo de carga, que se desplaza sobre orugas.

La unión entre la excavadora y el carro, cuyo papel recuerda más o menos el de una tolva, se halla asegurada por una cinta transportadora sobre pórtico, el cual puede deslizar sobre correderas para facilitar, dentro de amplios márgenes, el desplazamiento independiente de los dos elementos.

La excavadora de rueda de cangilones puede trabajar en pendiente o en zanja.

Las dos posibilidades se complementan y su suman, aumentando las facetas de excavación.

La profundidad del trabajo en zanja puede representar el $50 \%$ de la altura de avance en pendiente.

El abatimiento del brazo porta-rueda, bajo el nivel del suelo sobre el que apoya la excavadora, ha sido posible gracias a un dispositivo complementario, el cual permite alcanzar pendientes de $35^{\circ}$ a $40^{\circ}$ de inclinación con la horizontal.

En lo que respecta al funcionamiento de la máquina, la elección de la velocidad de giro de la rueda es función de la naturaleza y compacidad de los materiales a excavar.

Cuando estos últimos son duros, la velocidad de giro será elevada, lo cual viene en detrimento eventual del rendimiento máximo teórico o admisible.

Doblando la velocidad, y suponiendo constante el espesor del terreno que toma cada cangilón, el rendimiento queda sensiblemente reducido, lo que significa descenso del rendimiento de corte por cada vuelta de la rueda.

Podemos deducir, pues, que cuando se trate de excavar materiales duros no es necesario dar a la rueda dimensiones y potencia excesivas.

En razón de su gran velocidad, la rueda actúa más bien como una fresa.

Este caso se presentó cuando la cuchara de una pala mecánica no podía excavar una formación geológica muy dura sin recurrir a la disgregación previa mediante explosivos, mientras que la rueda de cangilones "mordía" muy bien sin intervención de este explosivo.

La forma de la cuchara y el ángulo de ataque del filo presentan una gran importancia desde el punto de vista del esfuerzo de corte y facilidad de vertido (evitando la colmatación de las cha. pas), respectivamente.

En consecuencia, la forma queda determinada for el tipo de material a excavar. Se buscan formas redondeadas, con objeto de evitar la adherencia de los materiales arcillosos.

Los cangilones pueden llevar una cinta de refuerzo con borde cortante o tener elementos de corte fijos en las cucharas para facilitar la penetración y el corte de la arcilla.

El desgaste mínimo de los cangilones se obtiene cuando la sección del corte en el terreno es cuadrada; no obstante, cuando el material es resistente, el esfuerzo de corte puede disminuir, dando pequeñas pasadas, en las cuales los elementos de corte disgregan el terreno antes de que el borde del cangilón penetre en la masa para llenarse.

Desde hace 20 años el volumen de las cucharas podía alcanzar $4 \mathrm{~m}^{3}$, girando a una velocidad periférica de $4 \mathrm{~m} / \mathrm{s}$, correspondiente a la velocidad de la cinta transportadora.

El vertido de los materiales sobre la cinta necesita ciertas precauciones para prevenir su dete. rioro. 
El primer impacto lo recibe una superficie intermedia, y la cinta en este punto va soportada por rodillos amortiguadores muy próximos y revestidos de caucho ligero.

De esta forma quedan disminuidos los efectos de impacto de los materiales que caen continuamente sobre la cinta.

En ciertos sectores del Valle de Colonia la extracción del lignito necesita separar las tierras estériles de un espesor de $150 \mathrm{~m}$. Las excavadoras de rueda de cangilones han sido estudiadas para $10.000 \mathrm{~m}^{3} / \mathrm{h}$, o incluso más.

En explotaciones similares, siempre a cielo abierto, se han comparado potentes dragalinas, con rueda de cangilones de capacidad análoga. A igualdad de peso de material, el rendimiento es ampliamente superior (hasta un $80 \%$ más) al segundo tipo de máquina, con una potencia instalada netamente inferior.

Cuando se prestan las condiciones del yacimiento, y en particular cuando es necesario rechazar las capas estériles, a más de $100 \mathrm{~m}$, la elección no admite dudas, sobre todo si hay grandes alturas de terreno a atacar y a desplazar.

La construcción de excavadoras de rueda de cangilones es, desde su origen, una especialidad alemana: LAUCHHAMMER MITTELDEUTSCHE, GESELLCHAFT, LÜBECK, DEMAG y, sobre todo, KRUPP INDUSTRIE und STAHLBAU.

Citemos, por ejemplo, en U.S.A., la firma BUCKEYE GARWOOD-CLEVELAND, aunque los americanos siguen prefiriendo las dragalinas y palas gigantes para los grandes movimientos de tierras. En el curso de los últimos años, KRUPP ha puesto a punto una serie de excavadoras de rueda de cangilones, tipo standard, para pequeños y medios rendimientos tipo C.100.

Aunque construidas de una forma compacta, estas máquinas son, sin embargo, fácilmente desmontables para su transporte.

Pueden ser concebidas con un generador Diesel, que produce, de una forma autónoma, energía eléctrica, y trabajar con una instalación de transporte deslizante.

Un montaje de este tipo trabaja en una explotación de lignito de la R.F.A., a cielo abierto, y en Fosfatos de Togo, sobre un parque de almacenamiento. El tipo C.300, un poco más potente, está en servicio en Australia, Japón y Togo (fig. 4).

Las dos mayores excavadoras de rueda de cangilones de la KRUPP, de tipo standard (C.500), se emplean en la extracción de tierras de recubrimiento y de lignito en Neyveli (India). Son especialmente indicadas para suelos duros, y los cangilones de la rueda van provistos de dientes revestidos de metal duro (carburo de tungsteno o metal de desgaste widia).

Debido a su gran movilidad, varias excavadoras de rueda de cangilones, tipo standard, se utilizan como medios auxiliares en las explotaciones a cielo abierto, para la construcción de carreteras, para la excavación de zanjas, o para trabajos de mejora del suelo, dando resultados satisfactorios.

La figura 5 corresponde a las máquinas en servicio en las minas italianas de lignito a cielo abierto de TRASIMENO y SANTA BARBARA.

Seis excavadoras de rueda aún más potentes ran abierto recientemente la nueva explotación a cielo abierto de KARDIA, para producir anualmente 6 millones de toneladas de lignito.

La instalación moderna de ARJUZANX (Landes), de KRUPP, para ELECTRICITÉ DE FRANCE, puede teóricamente producir hasta $3.600 \mathrm{~m}^{3} / \mathrm{h}$ con nueve cangilones de $1 \mathrm{~m}^{3}$ cada uno. 
La figura 6 presenta las principales características. El brazo alcanza una altura total de $40 \mathrm{~m}$ de frente, con cintas transportadoras en artesa de $1.800 \mathrm{~mm}$ de ancho y velocidad de 3,5 a 4,1 metros por segundo. La excavadora alimenta de lignito a la central térmica de MORCENX.

El peso de la máquina en servicio alcanza $2.700 \mathrm{t}$ y una potencia instalada de $2.400 \mathrm{~kW}$.

Para la explotación de lignito del valle de ROVINARI (Rumania), KRUPP ha proporcionado tres máquinas con una capacidad unitaria de $40.000 \mathrm{~m}^{3} /$ dia de gangas.

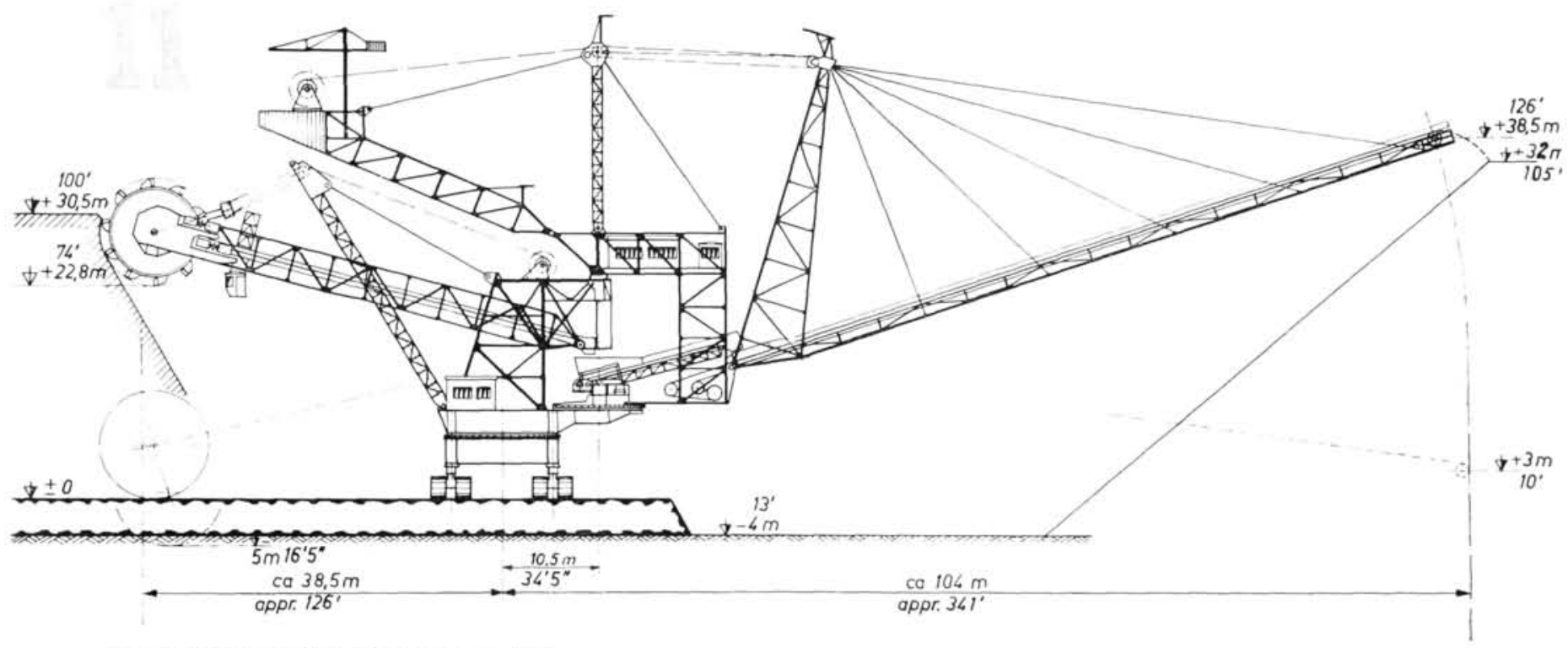

PEABODY COAL Co. (San Luis, U.S.A.)

Capacidad de excavación: $112.000 \mathrm{~m}^{3} /$ dia.

PEABODY COAL Co.

(San Luis, U.S.A.).

Capacidad de excava.

ción: $112.000 \mathrm{~m}^{3} / \mathrm{dia}$.

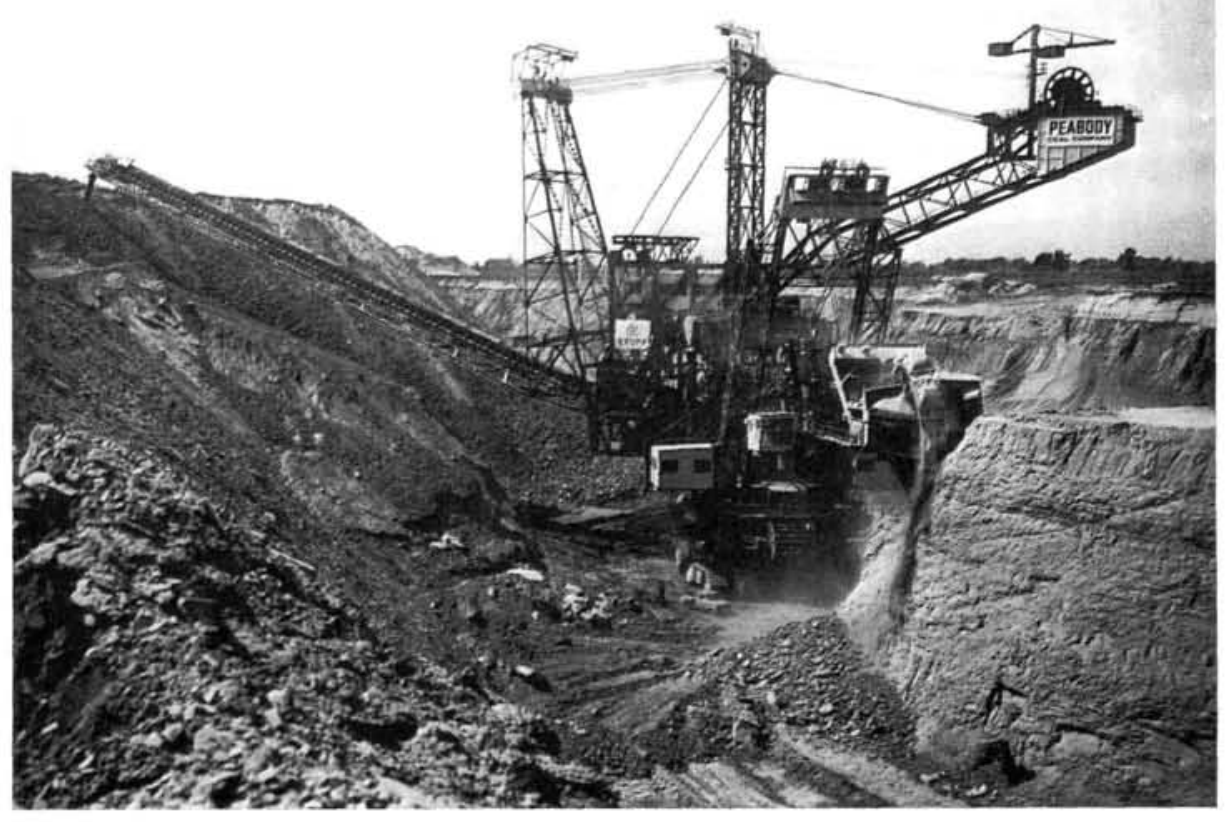


La rueda tiene un diámetro de $11,5 \mathrm{~m}$ y lleva nueve cangilones de $1,4 \mathrm{~m}^{3}$. La potencia de arrastre de la única rueda es de $630 \mathrm{~kW}$.

En Australia trabajan dos excavadoras de rueda de cangilones en las minas a cielo abierto de MORWELL y de YALLOURN, en los alrededores de MELBOURNE (State Electricity Commission of Victoria).

Estas máquinas son de avance, es decir, que el brazo porta-rueda puede desplazarse $9 \mathrm{~m}$ con la excavadora.

La regulación del corte se efectúa por avance de la rueda excavadora y no, como es tradicional, por desplazamiento del conjunto de la máquina sobre orugas.

La rueda, de un diámetro de $8,80 \mathrm{~m}$, lleva nueve cangilones de $1 \mathrm{~m}^{3}$ y gira a 3 r.p.m., lo que da aproximadamente 27 descargas de cangilón por minuto.

La velocidad de corte varía entre 1,18 y $1,54 \mathrm{~m} / \mathrm{s}$, lo que corresponde a una producción teórica de 1.380 a $1.800 \mathrm{~m}^{3} / \mathrm{h}$ por cada máquina.

Desde hace más de 2 años funciona en MORWELL una tercera excavadora para la misma central eléctrica australiana.

Dos máquinas de una potencia superior $\left(60.000 \mathrm{~m}^{3} / \mathrm{dia}\right)$ aseguran la explotación de lignito y la extracción de tierras estériles en FRIMMERSDORF y ZUKUNFT, en el valle del Rin (figs. 7 y 8 ). Cada una de ellas puede producir $5.270 \mathrm{~m}^{3} / \mathrm{h}$, con un diámetro de la rueda de $12,25 \mathrm{~m}$ y diez cangilones de $2 \mathrm{~m}^{3}$ cada uno. Se cuenta, pues, con 44 vertidos por minuto. La potencia de arrastre es de $720 \mathrm{~kW}$.

Las cintas transportadoras, en artesa, tienen $2,20 \mathrm{~m}$ de ancho, con una velocidad de $4 \mathrm{~m} / \mathrm{s}$.

Cada máquina va sobre tres orugas, desplazándose, según las exigencias de producción, de 3 a 8 metros por minuto.

La presión específica media sobre el suelo no sobrepasa los $0,82 \mathrm{kp} / \mathrm{cm}^{2}$.

Otra creación de modelo gigante (figs. 9 y 10) presenta las siguientes características:

Producción de tierras en la mina de GARSDORF, $112.000 \mathrm{~m}^{3} /$ día.

Puede excavar hasta $52 \mathrm{~m}$ por encima y $25 \mathrm{~m}$ por debajo del plano de rodadura de las orugas, y a $16 \mathrm{~m}$ por encima y $14 \mathrm{~m}$ por debajo de la instalación de transporte de los materiales excavados.

La excavación es, pues, posible sobre un frente total de $107 \mathrm{~m}$. Para la excavación en profundidad (hasta $25 \mathrm{~m}$ ) se ha incorporado al brazo porta-rueda una instalación de trans. porte con pendiente rígida.

La cinta transportadora está recubierta con una segunda cinta reforzada, de manera que es posible subir los materiales con una inclinación de $36^{\circ}$ con la horizontal.

La explotación de FRIMMERSDORF (R.F.A.), mencionada anteriormente, ha añadido en los últimos meses a su parque de excavadoras el potente modelo de $112.000 \mathrm{~m}^{3} /$ día.

Para una explotación a cielo abierto en U.S.A. (PEABODY COAL Co., en San Luis), KRUPP ha puesto a punto una excavadora de rueda para el vertido directo de los materiales excavados. 
Fresadora cargadora WESTFALIA “Tejón” (Westfalia Lünen, R.F.A.).

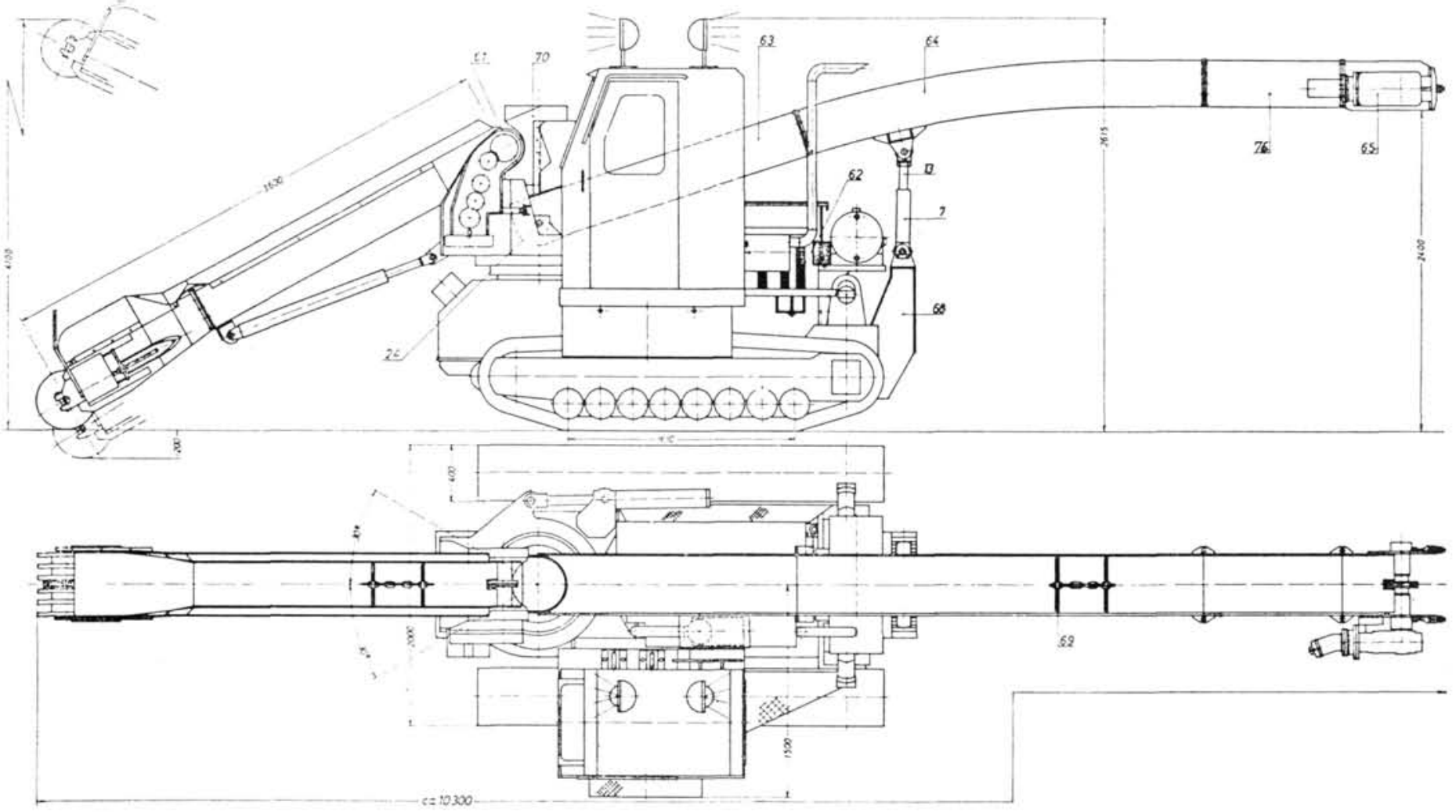

Tipo de excavadora "Tejón" trabajando en cantera de arcilla (Westfalia Lünen, R.F.A.).

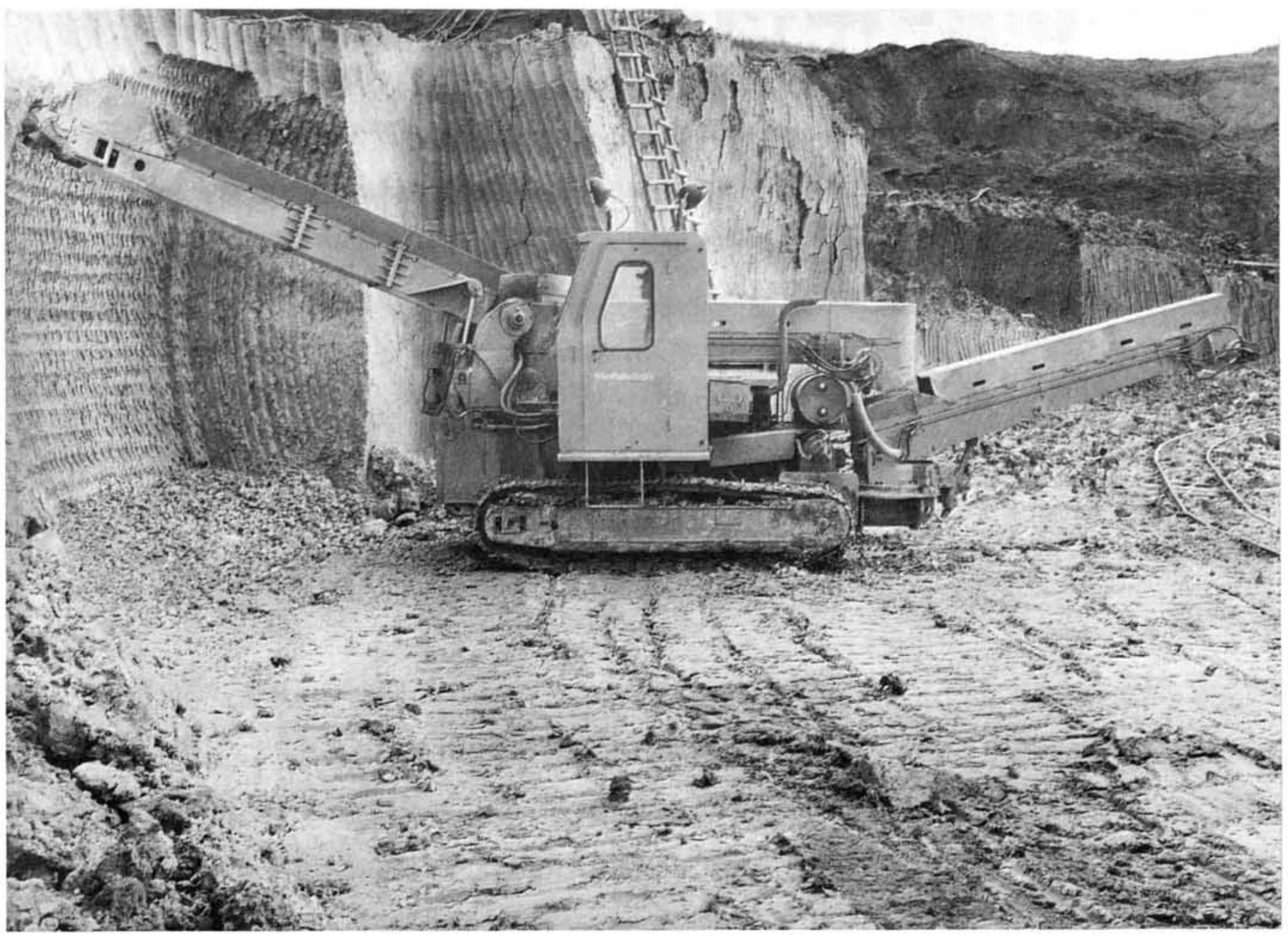


En la figura 11 se reproducen, en un dibujo, las características principales, y en la figura 12 se aprecia el conjunto de la máquina.

Debemos subrayar las principales magnitudes:

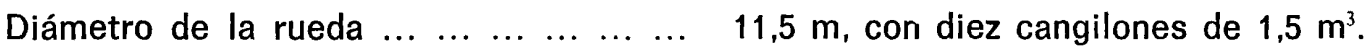

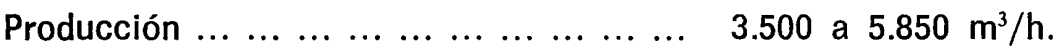

Potencia de arrastre de la rueda $\ldots \ldots \ldots . . .1 .160 \mathrm{~kW}$.

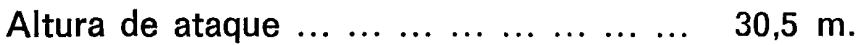

Altura total de la máquina $\ldots \ldots \ldots \ldots . . . . . \quad 37 \mathrm{~m}$.

Ancho de las cintas transportadoras ... ... 2,20 m, con una velocidad de $4 \mathrm{~m} / \mathrm{s}$.

Velocidad de traslación de las orugas ... $6 \mathrm{~m} /$ minuto.

Potencia total instalada ... $\ldots \ldots \ldots \ldots \ldots \ldots .4,325 \mathrm{~kW}$.

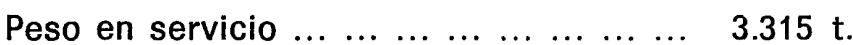

En Polonia, la extracción de gangas de recubrimiento de las capas de azufre sedimentario se realiza con la excavadora de rueda de cangilones.

En Suiza, los resultados obtenidos en una gravera han demostrado que una explotación continua es más económica que la explotación por medios tradicionales.

Anteriormente se extraía una grava compacta, de una granulometría de hasta $300 \mathrm{~mm}$, por medio de una pala mecánica, y se evacuaba con camiones. Esta pala, de $1 \mathrm{~m}^{3}$ de capacidad y de $30 \mathrm{t}$ de peso, no podía soportar más de una producción de $40 \mathrm{~m}^{3} / \mathrm{h}$, y la utilización de una pala de mayores dimensiones no parecía ofrecer demasiadas ventajas.

Desde hace 2 años se ha reemplazado esta pala mecánica por una excavadora de rueda del tipo 100 , evacuándose los materiales con cintas transportadoras en lugar de los camiones. La explotación se hace de forma continua.

La excavadora KRUPP dispone de un grupo Diesel de $277 \mathrm{CV}$. Está montada sobre un tren de orugas y puede excavar de 200 a $250 \mathrm{~m}^{3} / \mathrm{h}$.

Combinada con topadora producen conjuntamente de 300 a $350 \mathrm{~m}^{3} / \mathrm{h}$, trabajando la topadora ante los cangilones en la zona cuya posición en altura queda fuera del alcance de éstos.

Se pueden multiplicar los ejemplos de este tipo.

En trabajos subterráneos la excavadora de rueda aporta una solución interesante y económica, siempre que el terreno a excavar no sea demasiado cimentado o silicoso, es decir, demasiado compacto y duro.

Con este fin es posible construir excavadoras de dimensiones reducidas para no obstaculizar el resto de los trabajos.

Las condiciones particulares de explotación implican la excavación de débiles espesores y con galerías de gálibo limitado, lo que conduce a yuxtaponer dos excavadoras de rueda a fin de obtener, desde los dos lados, pequeños ángulos de evacuación. 
Siguiendo el sentido de posición, trabaja una sola rueda. Las dos excavadoras vierten sobre una cinta común de transporte. La descarga de tierras sobre la cinta transportadora se efectúa en el centro de la zona de situación.

La máquina obstaculiza la galeria menos que cualquier otra de excavación, perforación o socavación.

Una faceta particular de las excavadoras de rueda de cangilones es el trabajo en stocks de carbón o materiales pulverulentos, tales como: fosfatos, cemento, azufre, etc.

En Marruecos ha sido concebida una instalación, realizada por DEMAG LAUCHHAMMER, para permitir el almacenamiento inmediato y la carga de fosfato húmedo. Está compuesta de una excavadora de rueda de $825 \mathrm{t} / \mathrm{h}$ de producción, de un aparato de descarga (producción: $3.000 \mathrm{t} / \mathrm{h}$ ) y de un sistema de cintas transportadoras con carros de alimentación y transporte de $2.700 \mathrm{~m}$ de largo, cuyos anchos de correa varían de 1 a $1,4 \mathrm{~m}$.

La alimentación se hace por medio de una cinta transportadora fija, de un carro-transportador y de una cinta desplazable con carro de evacuación y máquina cargadora.

La toma de materiales se efectúa a base de la rueda, con cintas desplazable y fija de evacuación.

Las cintas transportadoras desplazables eran independientes en razón de la gran capacidad del stock, que puede alcanzar la extraordinaria cifra de 5 millones de metros cúbicos.

En la nueva fábrica de cemento en SOUSELA (Portugal), cerca de Coimbra, la caliza se tritura en una machacadora, de doble rotor, que produce $450 \mathrm{t} / \mathrm{h}$.

La caliza reducida se almacena en un parque de homogeneización capaz para 24.000 t. Este parque está dotado de un aparato para el vertido de $450 \mathrm{t} / \mathrm{h}$, y de una rueda de cangilones de $250 \mathrm{t} / \mathrm{h}$ para la carga.

En Lacq se utiliza una rueda para la carga de azufre solidificado desde un stock. Dada la forma de los cristales de azufre, la topadora resulta inadecuada, ya que patinan las cadenas y existe el peligro de incendio por recalentamiento.

La firma WESTFALIA-LÜNEN ha creado una fresa-cargadora (realmente, máquina de excavación) con tambor-cortante y para carga, que puede trabajar en terrenos de dureza media. Este modelo reducido recuerda la idea de una rueda de cangilones. Los materiales son extraídos del macizo (figs. 13 y 14) por un tambor de corte, que no lleva cangilones, sino picos. El tambor cortante va montado sobre un brazo móvil y es arrastrado por la cadena de este brazo.

Los materiales se vierten sobre una cinta transportadora intermedia fija, y después sobre la cinta del brazo orientable posterior. Más allá de este punto, la evacuación se puede organizar con otros transportadores, camiones o vagones.

La elevación del brazo de corte es posible hasta $4,1 \mathrm{~m}$ por encima, y $200 \mathrm{~mm}$ por debajo del suelo, con barrido lateral del macizo, por la fresa, de un ancho de 3,5 $\mathrm{m}$.

La longitud del brazo de corte es de $3,6 \mathrm{~m}$, pero puede ser alargado al construir la máquina.

Alimentado con un motor Diesel de $96 \mathrm{CV}$, o eléctrico de $90 \mathrm{~kW}$, posee transmisión hidráulica potente.

La superficie de las cadenas está regulada para que la presión sobre el suelo no pase de $0,82 \mathrm{kp} / \mathrm{cm}^{2}$. Ello permite un esfuerzo de empuje suficiente de la máquina contra el frente de ataque. 
résumé

Les excavateurs à roue-pelle dans les grands terrassements

Georges Viê, ingénieur des mines

L'auteur fait un historique de ce type d'engins et parle de leurs progrès considérables en taille, poids et efficacité au cours des trente dernières années, ajoutant que les projets les plus récents tendent à les placer dans une situation privilégiée, sans une concurrence apparente sur le marché, pour leur capacité de rendement et d'attaque des formations les plus dures.

Dans cet article, il décrit également le parties et les caractéristiques de ces exca vateurs et cite une série de leurs fabricants.

\section{summary}

Bucket Wheel Excavators for Large Earth Movements

Georges Vié, mining engineer

The author of this article gives a brief history of this kind of machine and discusses the considerable progress made in the last thirty years as to size, weight, and efficiency. Due to unique applicability to special projects, enormous yield, and capacity to attack extremely hard formations, these excavators enjoy a relatively unchallenged market position.

Described here are the different types, component parts, workings, important characteristics, and current manufacturers of these machines.

\section{zusammenfassung}

Eimerbagger bel grossen Erdbewegungen

Georges Vié, Bergbauingenieur

Der Autor gibt einen kurzen Oberblick auf die Geschichte dieses Maschinentyps und spricht über seine beachtlichen Fortschritte in den letzten 30 Jahren in bezug auf Grösse, Gewicht und Leistung. Er fügt hinzu, dass sie neuerdings eine bevorzugte Stellung einnehmen und keine bedeutende Konkurrenz auf dem Markt haben dank ihrer Leistung und ihrer Einsatzfähigkeit bei härtester Bodenbeschaffenheit.

In diesem Artikel werden die Einzelteile und deren Funktionieren beschrieben, sowie die Hersteller dieser Maschinen, die Typen und thre besonderen Merkmale genannt.

\section{publicación del i.e.t.c.c.}

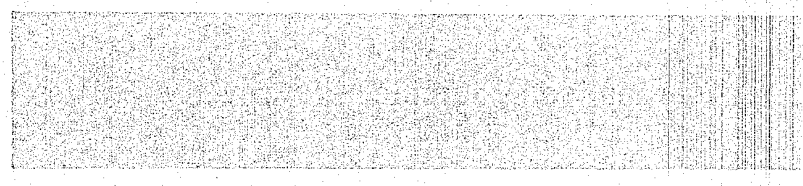

importancia creciente que se concede a la protección química de las obras de fábrica y la carencia de un tratado que reúna toda la literatura dispersa que existe sobre el tema, han Ilevado a la publicación de este libro, que debe leerse con atención en todas y cada una de sus páginas a fin de que nada resulte incomprensible.

En la Technische Akademie de Wuppertal el autor celebró, durante los últimos años, ocho seminarios, de tres días de duración cada uno, sobre protección de obras. Los participantes a

\section{protección química de la construcción}

\author{
HANS KöLZOW
}

Dr. Químico Dipl. Stadtbaurat a.D.

estos seminarios han sido arquitectos $e$ ingenieros procedentes de organismos oficiales y de empresas privadas, así como químicos de las industrias dedicadas a la fabricación de sistemas y métodos químicos protectores. De las conferencias y de los coloquios consiguientes se han obtenido resultados importantes y esperanzadores que merecen sor conocidos por círculos mucho más amplios. Por todo ello se ha creído conveniente la publicación del presente resumen, indicando expresamente que, de ningún modo, se trata de un manual ni de un libro de recetas.

Encuadernada en rústica, de $17 \times 24 \mathrm{~cm}$, compuesto de 74 páginas. Madrid, 1971.

Precios. España, 300 pesetas. Extranjero, \$ 6 . 